\title{
Curcumin-Loaded Nanoparticles Protect Against Rhabdomyolysis-Induced Acute Kidney Injury
}

\author{
Xiaoling Chen ${ }^{\mathrm{a}}$ Jian Sun ${ }^{\mathrm{b}}$ Hailun Li ${ }^{\mathrm{a}}$ Hongwu Wang ${ }^{\mathrm{a}}$ Yongtao Lin ${ }^{\mathrm{c}} \mathrm{Yu} \mathrm{Hu}$ \\ Donghui Zhenga \\ aDepartment of Nephrology, The Affiliated Huaian Hospital of Xuzhou Medical University, Huai'an, \\ 'Department of Anesthesiology, Huai'an Maternal and Child Health Hospital, Huaian, ' Jiangsu \\ Vocational College of Nursing, Huai'an, China
}

\section{Key Words}

Rhabdomyolysis (RM) • Acute kidney injury (AKI) • Curcumin • Anti-oxidant • Nanoparticles

\begin{abstract}
Background/Aims: Rhabdomyolysis (RM) is a potentially life-threatening condition that results from the breakdown of muscle and consequent release of toxic compounds into circulation. The most common and severe complication of RM is acute kidney injury (AKI). This study aimed to evaluate the efficacy and mechanisms of action of curcumin-loaded nanoparticles (Cur-NP) for treatment of RM-induced AKI. Methods: Curcumin-NP was synthesized using the nanocarrier distearoylphosphatidylethanolamine-polyethylene glycol (DSPE-PEG) to achieve a prolonged and constant drug release profile compared with the curcumin-free group. The anti-AKI effects of Curcumin-NP were examined both in vitro (myoglobin-treated renal tubular epithelial HK-2 cells) and in vivo (glycerol-induced AKI model). Results: Our results indicated that Curcumin-NP reversed oxidative stress, growth inhibition and cell apoptosis accompanied with down-regulation of apoptotic markers Caspase-3 and GRP-78 in vitro. In vivo studies revealed enhanced AKI treatment efficacy with Curcumin-NP as characterized by reduced serum creatine phosphokinase (CPK), creatinine $(\mathrm{Cr})$ and urea and less severe histological damage in renal tubules. In addition, kidney tissues from Curcumin-NP-treated AKI rats exhibited reduced oxidative stress, apoptosis, and cleaved Capase-3 and GRP-78 expression. Conclusion: Our results suggest that nanoparticle-loaded curcumin enhances treatment efficacy for RM-induced AKI both in vitro and in vivo.
\end{abstract}

\section{Introduction}

Rhabdomyolysis (RM) refers to the breakdown of muscle, resulting in release of potentially toxic compounds into the circulation, which causes damage to different tissues $\mathrm{X}$. Chen, J. Sun and H. Li contributed equally to this work. 


\section{Cellular Physiology Cell Physiol Biochem 2017;43:2143-2154

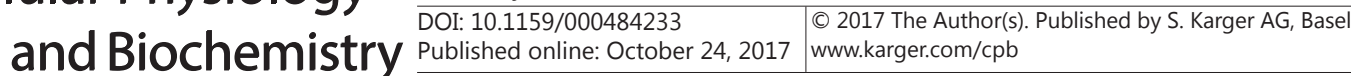 \\ Chen et al.: Curcumin-Loaded Nanoparticles Protect Against RM-Induced AKI}

and organs [1]. RM results from direct and indirect causes, such as crush injury, medications, infections, myopathies, muscular dystrophies, and various other diseases [2]. In Haff disease, the development of RM within 24 hours following ingestion of shellfish and certain freshwater fishes, has also been reported worldwide [3]. However, the exact pathogenesis of RM remains unclear, and RM treatment and care are limited.

Acute kidney injury (AKI) is a complex clinical syndrome with an abrupt loss of kidney function, which is a strong and independent risk factor for mortality. AKI is associated with an 8- to 10-fold increased death rate of hospitalized patients [4]. AKI is a common and the most severe complication of RM. Melli et al [5]. reported that in 475 hospitalized rhabdomyolysis patients, $218(46 \%)$ cases developed AKI. In addition, RM is the cause of renal failure in 5-25\% of all AKI cases [5]. In addition, patients with AKI are more likely to develop progressive chronic kidney disease (CDK), including end-stage renal disease (ESRD) [6]. Despite the application and development of a variety of new drugs and surgery, the incidence of AKI has increased over the past decade [7]. Although the pathogenesis and tissue damage of RM is a complex process, the accumulation of oxidative injury and increased cell apoptosis appear to be the end results of most causes of RM-induced AKI [7].

Curcumin (Cur), a hydrophobic polyphenolic compound derived from the rhizome of the herb curcuma longa, possesses a wide range of biological functions, including antioxidant activity $[8,9]$. Curcumin can effectively protect cultured renal tubular epithelial cells against oxidative stress induced by $\mathrm{H}_{2} \mathrm{O}_{2}$ and $\mathrm{LOOH}[10,11]$, suggesting that curcumin can be a potential therapy for RM-induced AKI. Despite curcumin's potential medicinal benefits, its application in the clinic is limited due to its poor aqueous solubility and low in vivo bioavailability. To address this obstacle, we hypothesize that a nano drug delivery system, namely, Curcumin-loaded nanoparticles (Cur-NP), exhibits advantages of high permeability and retention that can increase curcumin's bioactivity.

In this study, we synthesized Cur-NP using the nanocarrier distearoylphosphatidyl ethanolamine-polyethylene glycol (DSPE-PEG) and explore its therapeutic effect on RMinduced AKI both in vitro (myoglobin-treated renal tubular epithelial cell line, HK-2) and in vivo (glycerol-induced AKI model). Our results indicated that Cur-NP enhanced curcumin's anti-oxidant and anti-apoptosis effect both in vitro and in vivo and ameliorated the severity of AKI in rats. These results suggest that this Cur-NP delivery system may be a promising therapeutic for RM-induced AKI in the future.

\section{Materials and Methods}

\section{Cell culture and cell line}

The human renal tubular epithelial cell line HK-2 (purchased from the ATCC) was cultured in 50\% Dulbecco's modified Eagle's medium (DMEM) (HyClone, USA) and 50\% F12 supplemented with 10\% fetal bovine serum (FBS) (HyClone, USA), $100 \mathrm{U} / \mathrm{mL}$ penicillin (Gibco, Invitrogen) and $100 \mathrm{U} / \mathrm{mL}$ streptomycin (Gibco, Invitrogen) at $37^{\circ} \mathrm{C}$ with $5 \% \mathrm{CO}_{2}$.

\section{Preparation of curcumin-loaded nanoparticles (Cur-NP)}

Cur-NP were prepared using a previously described method with minor modifications [12]. Briefly, both DSPE-PEG (Ruixi Biological Technology, China) and curcumin (purity > 95\%) (Sciphar, China) solutions were prepared by dissolving in tetrahydrofuran (THF) at concentrations of $4.0 \mathrm{mg} / \mathrm{mL}$. After sonication to ensure that the solution was completely dissolved, curcumin solution was gently dripped into the DSPE-PEG solution (1:1) and pipetted repeatedly to mix thoroughly. For sonication, the mixed solution was diluted 10fold in double distilled water. The loading of the curcumin on nanoparticles was completed after 6 minutes of continuous sonication. The synthesized Cur-NP was covered with tin foil, and several holes were made on the lid to allow the THF to evaporate overnight. Cur-NP was loaded in a dialysis bag (molecular mass cut off is between 6-8 kDa) and incubated in $500 \mathrm{ml}$ double distilled water in a beaker. The water was changed twice daily and dialyzed overnight. The dialysis was completed when the water in the beaker was clear. The purified Cur-NP was freeze-dried, stored at $-20^{\circ} \mathrm{C}$ and kept away from light. 


\section{Cellular Physiology Cell Physiol Biochem 2017;43:2143-2154 \begin{tabular}{ll|l} 
DOI: 10.1159/000484233 & $\begin{array}{l}\text { O 2017 The Author(s). Published by S. Karger AG, Basel } \\
\text { www.karger.com/cpb }\end{array}$
\end{tabular}

\section{Transmission electron microscopy}

The morphology of Cur-NP was examined using a transmission electron microscope (TEM) JEM-100S (Jeol, USA). Nanoparticles were placed on copper grids covered with nitrocellulose membrane and stained using a $1 \%(\mathrm{w} / \mathrm{v})$ sodium phosphotungstate solution. Images were obtained after the sample was air-dried.

\section{Measurements of particle size and Zeta potential}

The measurements of particle size and Zeta potential were performed using a Particle Size Analyzer and Zeta Potential Analyzer (Briookheaven, USA), respectively. The measurement of Zeta potential was performed in double distilled water using disposable zeta cells and a general protocol at $25^{\circ} \mathrm{C}$. The instrument was calibrated routinely with a $-50 \mathrm{mV}$ latex standard. The mean zeta potential was determined using phase analysis light scattering technique.

\section{In vitro release of Cur-NP}

The dialysis dispersion method was employed to determine the in vitro release of curcumin from CurNP. Briefly, $10 \mathrm{mg}$ freeze-dried Cur-NP or Cur-free was dissolved in $1 \mathrm{ml}$ saline solution. Cur-NP or Cur-free were placed in dialysis bags (molecular mass cut-off is $12 \mathrm{kDa}$ ). The dialysis bags were incubated in 0.01 $\mathrm{mol} / \mathrm{L} \mathrm{PBS}\left(\mathrm{pH} \mathrm{7.4}\right.$ ) at $37^{\circ} \mathrm{C}$ with gentle shaking. At specific time points, the PBS was removed and replaced by the same amount of fresh PBS. The release of curcumin at different time points was determined using high-performance liquid chromatography (HPLC). Data are presented as the percentages of the released curcumin of the total curcumin in Cur-NP or Cur-free.

\section{In vitro rhabdomyolysis injury and drug intervention}

Myoglobin in the reduced state was prepared by dissolving $32.5 \mathrm{mg}$ vitamin $\mathrm{C}$ and $356 \mathrm{mg}$ myoglobin in $100 \mathrm{ml}$ DMEM. The solution was mixed thoroughly and then filtered through a $0.22-\mu \mathrm{m}$ filter. In the RM group, HK-2 cells were cultured in normal DMEM for $24 \mathrm{~h}$ and incubated with DMEM containing reduced myoglobin for an additional $24 \mathrm{~h}$. In the drug intervention group, cells were cultured in the myoglobincontaining DMEM in the presence of $50 \mu \mathrm{M}$ of Cur-free (RM+Cur group) or Cur-NP (RM+Cur-NP group) for $24 \mathrm{~h}$. Cells cultured in normal DMEM were considered as the control group.

\section{MTT Proliferation Assay}

HK-2 cells were seeded at a density of $1 \times 10^{4}$ per well in 96-well plate and divided into four groups. The groups received different treatments as described above. Twenty-four $\mathrm{h}$ after treatment, the medium was removed, and cells were washed with PBS. The MTT assay was performed according to the manufacturer's instructions using a commercial kit (Buke Biology, China). Briefly, $100 \mu$ l DMEM and $50 \mu$ l MTT were added into each well and pipetted up and down. After a 4-h incubation at room temperature, the plate was centrifuged at $1000 \mathrm{rpm}$ for $5 \mathrm{~min}$, and the supernatant was carefully removed. Then, $150 \mu \mathrm{DMSO}$ was added into each well and incubated at $37^{\circ} \mathrm{C}$ for $10 \mathrm{~min}$. Then, the plate was gently shaken for an additional $5 \mathrm{~min}$. Spectrometric absorbance at $490 \mathrm{~nm}$ was read using a microplate reader. Each experimental group was assessed in triplicate, and the experiment was repeated thrice.

\section{Analysis of cell apoptosis by flow cytometry}

After myoglobin and drug treatment, HK-2 cells were collected and washed twice with PBS. Then, cells were subjected to Annexin V and propidium iodide (PI) staining using an apoptosis assay kit (Beijing Baosai Biology, China) following the manufacturer's instructions. Flow cytometry was used to examine early stage apoptosis (Annexin $\mathrm{V}^{+} \mathrm{PI}$ ) and late stage apoptosis (Annexin $\mathrm{V}^{+} \mathrm{PI}^{+}$). The data were acquired by BD FACSCanto II (Becton Dickinson, USA) and processed using FlowJo software (Tree star, USA).

\section{Lipid peroxidation product Assay}

The amount of lipid peroxidation product malondialdehyde (MDA) was measured using a commercial MDA kit (Cell Biolabs, USA). The spectrophotometric absorbance was assessed at $532 \mathrm{~nm}$ in accordance with the manufacturer's instructions. The results were expressed as nmol MDA per mg protein.

Western blot

Cell lysates were prepared by suspending $1 \times 10^{6}$ cells in $150 \mu \mathrm{l}$ of RIPA lysis buffer (50 mM Tris, 150 $\mathrm{mM} \mathrm{NaCl}, 1 \%$ Triton X-100, 1\% sodium deoxycholate,0.1\% SDS, $100 \mathrm{mM}$ phenylmethylsulfonyl fluoride) 


\section{Cellular Physiology Cell Physiol Biochem 2017;43:2143-2154 \begin{tabular}{l|l|l} 
DOI: 10.1159/000484233 & $\begin{array}{l}\text { O 2017 The Author(s). Published by S. Karger AG, Basel } \\
\text { www.karger.com/cpb }\end{array}$
\end{tabular}

followed by centrifugation at $12,000 \mathrm{rpm}$ for $5 \mathrm{~min}$ at $4{ }^{\circ} \mathrm{C}$ to collect the supernatant. Then, $20 \mathrm{mg}$ of cell lysates were separated by $12 \%$ sodium dodecyl sulfate-polyacrylamide gel electrophoresis (SDS-PAGE) and then transferred onto nitrocellulose membranes. The membrane was probed with relevant primary antibodies and corresponding HRP-conjugated secondary antibodies. West Pico chemiluminescent (Thermo Fisher, USA) was used to visualize protein bands. The intensity of bands was quantified using ImageJ software. Normalization of specific proteins was performed based on GAPDH expression.

\section{Animals and treatment}

Eight-week-old male Sprague-Dawley rats were divided into four groups with 6 mice in each group. The Sham group was intramuscularly injected with PBS. The AKI model was established by administering an intramuscular injection of $50 \%$ glycerol $(10 \mathrm{mg} / \mathrm{kg})$ in the rats' limbs. Twenty-four $\mathrm{h}$ after glycerol injection, the AKI group received PBS, the AKI+Cur group received Cur-free $(10 \mathrm{mg} / \mathrm{kg}$, dissolved in PBS), and the AKI+Cur-NP group received Curcumin-DSPE-PEG (10 mg/kg, dissolved in PBS). All PBS, Curcumin and CurNP treatments were provided as a single dose and administered intravenously. Mice were sacrificed $72 \mathrm{~h}$ after initial glycerol injections. All animal studies were conducted in accordance with the principles and procedures outlined in the Huai'an Hospital of Xuzhou Medical University Guide for the Care and Use of Animals under assurance number SCXK (Jiangsu) 2008-0002.

\section{Renal function tests}

Serum $\mathrm{CPK}, \mathrm{Cr}$ and urea levels were determined using commercial kits (Cusabio, China) according to the manufacturer's instructions.

\section{Renal histopathology and TUNEL assay}

Rat kidney tissues were fixed in $10 \%$ neutral formalin and embedded in paraffin. Briefly, $3-\mu \mathrm{m}$ sections were stained with hematoxylin and eosin (H\&E). Photographs of the kidney cortex of each mouse were obtained from five random fields for analyzing the histopathology changes. The histopathological changes were evaluated by a pathologist in a blinded fashion according to the degree of tubular necrosis, hemorrhage and cast formation as follows: 0 , no change; 1 , change affecting less than $25 \%$ of the field; 2 , change affecting $25-50 \%$ of the field; 3 , change affecting $50-75 \%$ of the field; 4 , change affecting greater than $75 \%$ of the field [13]. The other half of the kidney samples was also assessed by transmission electron microscopy (Hitachi, Tokyo, Japan) under uranyl acetate and lead citrate double staining. For TUNEL assay, sections were stained according to the manufacturer's instructions of the commercially available kit. Photographs of the kidney cortex of each mouse were obtained from five random fields with $400 \times$ magnification. Photos were analyzed by Image pro-plus (6.0) to calculate the percentages of positive-stained cells/total cells in each field, representing the percentage of apoptosis.

\section{Statistical analysis}

Data were presented as the mean \pm standard deviation (SD) and analyzed using SPSS 13.0 statistical software (SPSS Inc., USA). Statistical analysis between two sets of data was performed using Student's t-test. $P<0.05$ was considered statistically significant.

\section{Results}

\section{Solubility of Curcumin and Curcumin-NPs}

Precipitation of Curcumin in water (Fig. 1A) indicated that Curcumin is poorly water soluble. The water solution of blank NPs is light blue and transparent, whereas that of Cur-NPs is yellow and transparent, suggesting that encapsulation in DSPE-PEG NPs enhanced the water solubility of Curcumin (Fig. 1C).

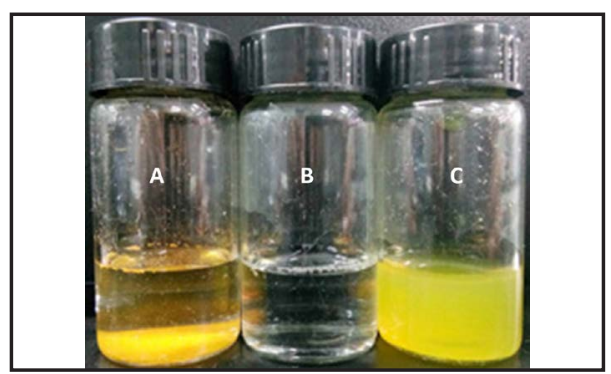

Fig. 1. The solubility of Cur-NPs in water. A. Non-encapsulated curcumin. B. Blank nanoparticles. C. Cur-NPs. 
Fig. 2. Characteristics of Curcumin-loaded nanoparticles. (A) TEM images depicting the regular spherical shape of Cur-NP nanoparticles. (B) Particle analysis revealing that the average diameter of Cur-NP is $80.29 \mathrm{~nm}$, and most of the particles range from 80$100 \mathrm{~nm}$ in diameter. (C) The Zeta potential of Cur-NP exhibit a negative value $(-5.91$ $\mathrm{mV}$ ). (D) Cur-NP and Cur-free were dialyzed in PBS. The amount of released Cur in the incubation medium was determined by HPLC at indicated time points. Data are presented as the percentages of the released curcumin of the total curcumin in Cur-NP or Cur-free.
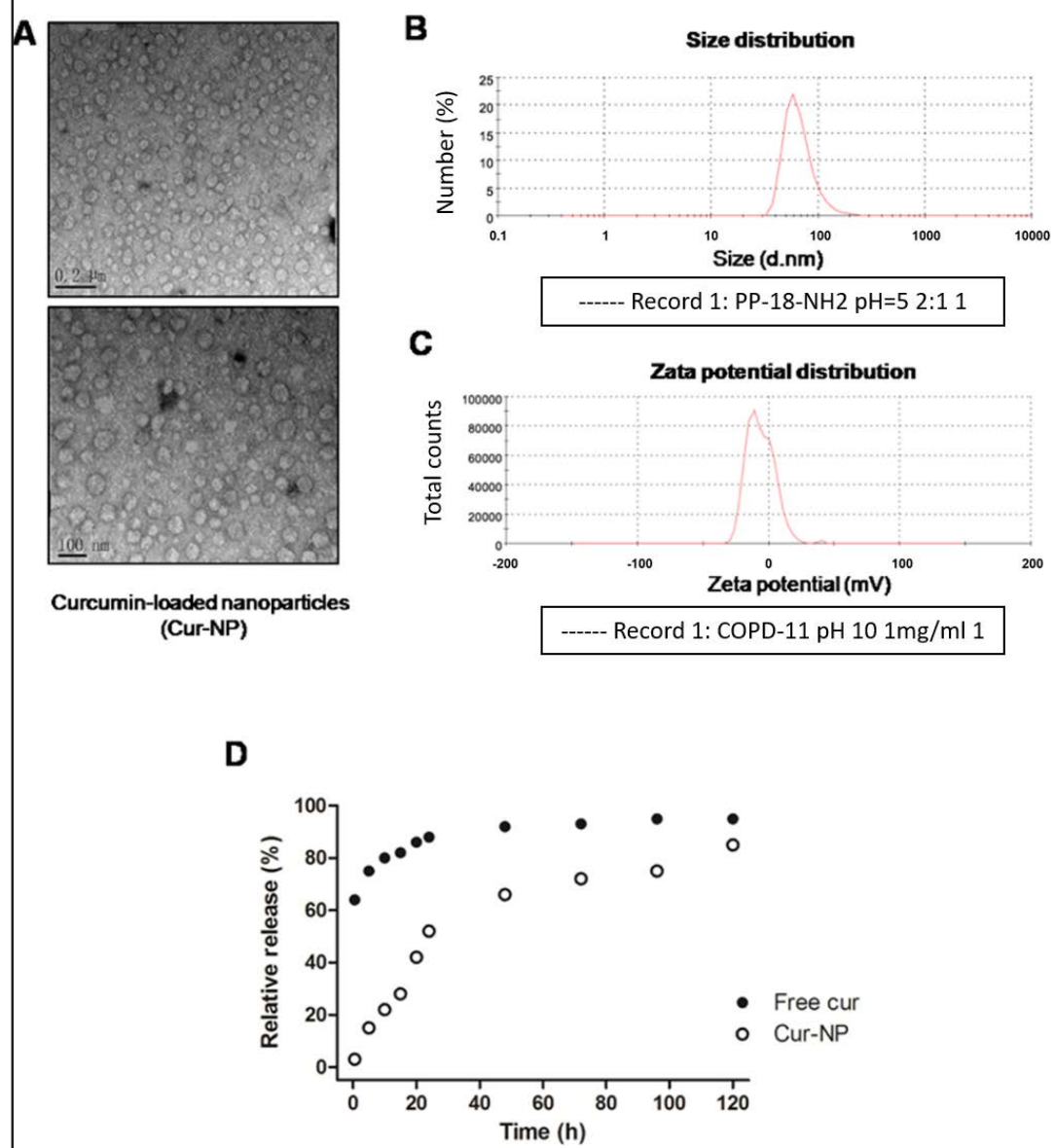

Characteristics of Curcumin-loaded nanoparticles

Curcumin-loaded nanoparticles (Cur-NP) were prepared as previously reported [12] and described above. In the present study, the nanomaterial distearoylphosphatidyethanolamin-polyethylene glycol (DSPE-PEG) was employed. As shown in the images obtained by transmission electronic microscopy (TEM), Cur-NP are 80 100 nm in diameter with a regular spherical shape (Fig. 2 A). As measured by the Particle Size Analyzer and Zeta Potential Analyzer (Briookheaven, USA), on average, Cur-NPs are $80.29 \mathrm{~nm}$ in diameter, and the Zeta potential exhibits a negative value $(-5.91 \mathrm{mV}$ ) (Fig. $2 \mathrm{~B}-\mathrm{C})$. To determine the release profile of Cur-NP, both Cur-free and Cur-NP was dialyzed using a dialysis bag with a $12 \mathrm{kDa}$ molecular weight cut-off. As expected, the release of Curcumin from Cur-NP solution was significantly slower than Cur-free. In the initial $20 \mathrm{~h}$ of dialysis, approximately $80 \%$ of Cur-free was released from the dialysis bag, whereas only $40 \%$ was released from Cur-NP in the same time period (Fig. 2 D). In the following $80 \mathrm{~h}$, Curcumin was gradually released from Cur-NP, and the total release reached $85 \%$ by the end of $120 \mathrm{~h}$ (Fig. $2 \mathrm{D}$ ). These results demonstrate that the majority of the loaded Curcumin was released from Cur-NP nanoparticles. The release profile is prolonged and constant compared with Cur-free.

\section{Curcumin-loaded nanoparticles inhibit myoglobin-induced renal cell injury in vitro}

The accumulation of oxidative stress is an important end cause of RM-induced AKI. As such, the anti-oxidative Curcumin can be a potential therapeutic for treating RM-induced AKI. We first determined whether Cur-NP exhibits an increased or at least a similar protective effect against RM-induced renal cell injury in vitro. To establish the RM-induced renal 
Fig. 3. In vitro anti-oxidative effect of Cur-NP. HK-2 cells were cultured with DMEM with (RM group) or without (Control Group) reduced myoglobin for $24 \mathrm{~h}$. In the drug intervention group, HK-2 cells were treated with reduced myoglobin in the presence of $50 \mu \mathrm{M}$ Cur-free (RM+Cur group) or Cur-NP
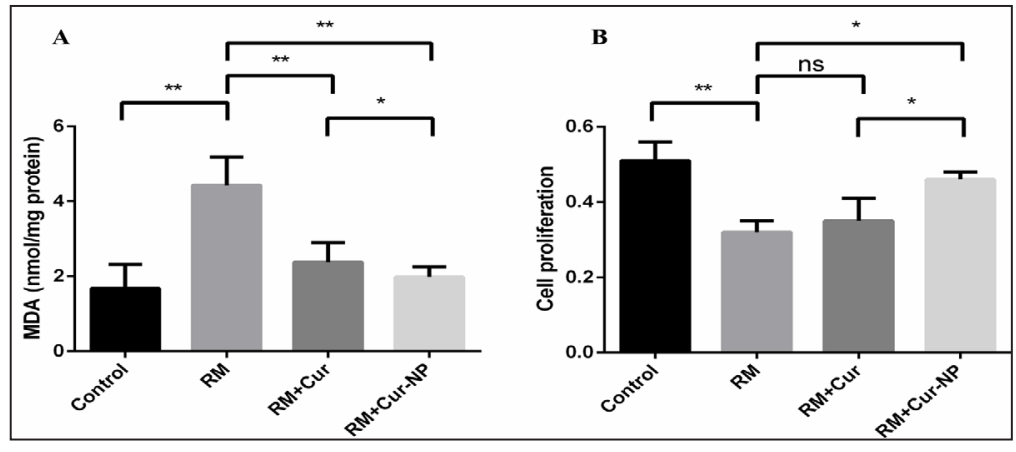

(RM+Cur-NP group) for $24 \mathrm{~h}$. (A) The levels of lipid peroxidant products in different groups as measured by the MDA assay. (B) Cell proliferation in different groups measured by the MTT assay. Data are presented as the mean \pm SD of each group. Statistical significance between two groups was determined by Student's t test. Significance levels are presented as indicated: ${ }^{*} \mathrm{P}<0.05$; ${ }^{* *} \mathrm{P}<0.001$.

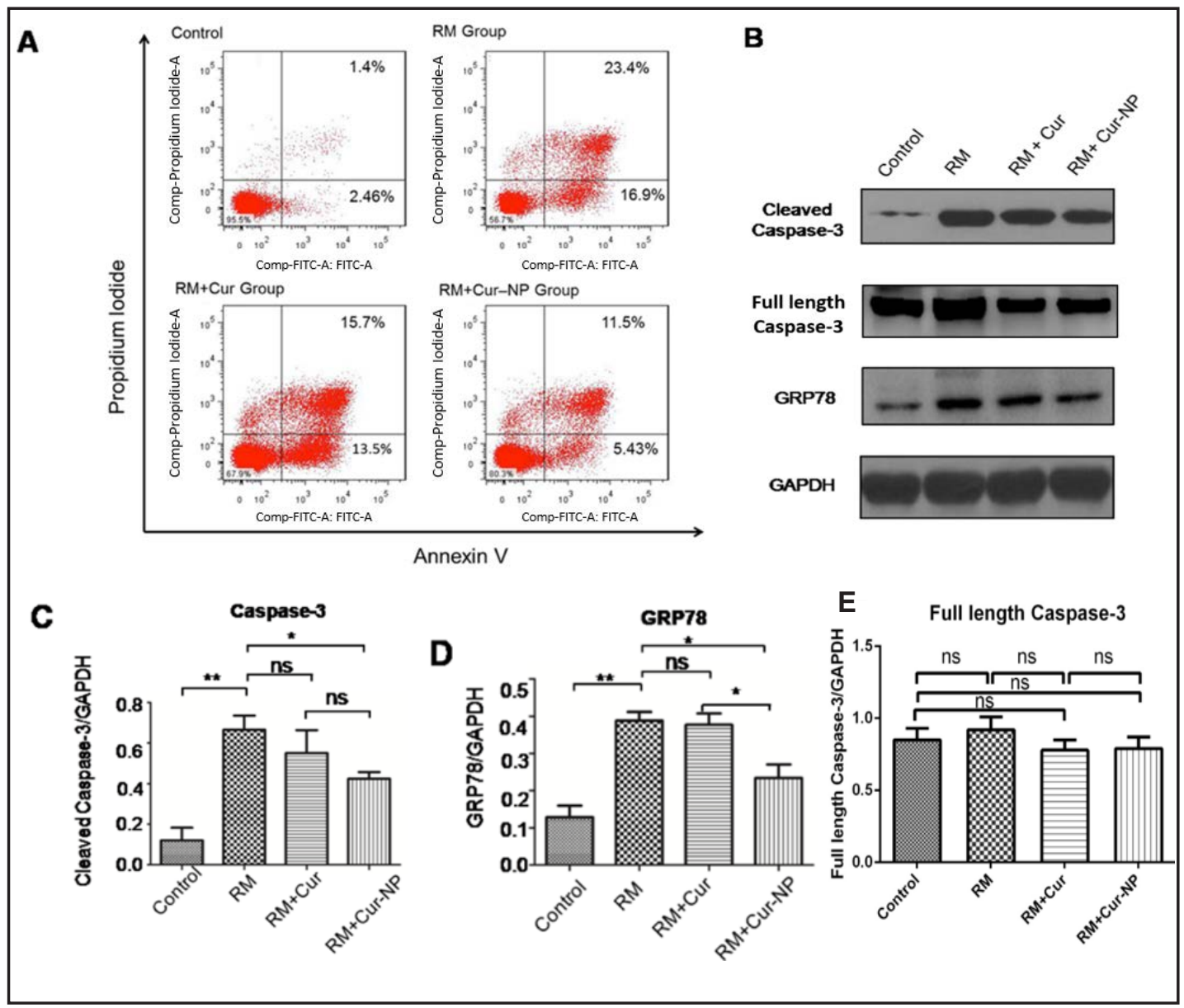

Fig. 4. The in vitro anti-apoptotic effect of Cur-NP. HK-2 cells were stimulated with reduced myoglobin and treated with Cur-free or Cur-NP as described above. (A) Twenty-four h following treatment, cells were harvested and stained with Annexin-V and PI and analyzed by flow cytometry. (B) Western blotting analysis of cleaved Caspase-3, full length Caspase-3, GRP78 and GAPDH expression in cells. (C and D) Quantitative analysis of Western blotting results. Specific protein expression levels were normalized to GAPDH levels. Data are presented as the mean \pm SD of three independent experiments. Statistical analysis between highlighted groups was determined by student's t-test. Significance levels were labeled as follows: ns $\mathrm{P}>0.05$; $^{*} \mathrm{P}<0.05 ;{ }^{* *} \mathrm{P}<0.01$. 


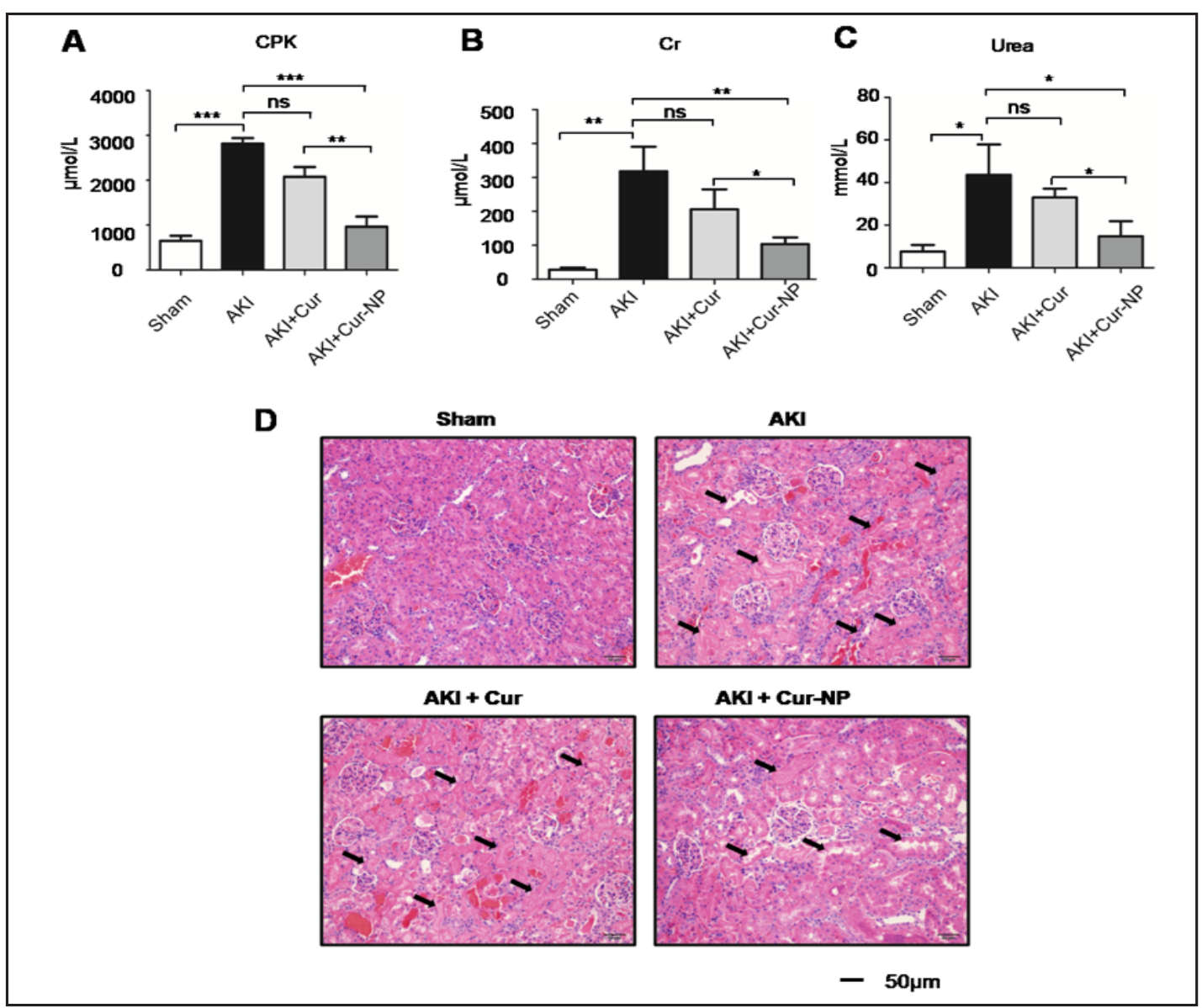

Fig. 5. Cur-NP protects against glycerol-induced AKI in rats. (A-C) Eight-week-old male Sprague-Dawley rats were divided into four groups ( $n=6 /$ group). The Sham group was intramuscularly injected with PBS. The AKI model was established by administering an intramuscular injection of $50 \%$ glycerol $(10 \mathrm{mg} / \mathrm{kg}) \mathrm{in}$ the limbs. Twenty-four $h$ after glycerol injection, the AKI group received PBS, the AKI+Cur group received Cur-free $(10 \mathrm{mg} / \mathrm{kg})$, and the AKI+Cur-NP group received Curcumin-DSPE-PEG (10 mg/kg). Rats were sacrificed $72 \mathrm{~h}$ post glycerol injection, and serum creatine phosphokinase (CPK) (A), creatinine (Cr) (B) and urea (C) levels in each group were examined after rats were sacrificed. Data are presented as the mean \pm SD of each group. Statistical significance between two groups was determined by Student's t test. Significance levels are presented as indicated: $\mathrm{ns} \mathrm{P}>0.05$; ${ }^{*} \mathrm{P}<0.05$; ${ }^{* *} \mathrm{P}<0.01$; ${ }^{* *} \mathrm{P}<0.001$. (D) Representative photos of H\&E sections of kidneys from each group. Scale bar: $50 \mu \mathrm{m}$. Cellular swelling and tubular epithelial necrosis are detectable in AKI rats (See arrows). Reduced areas of tubule damage are detectable in the AKI+Cur-NP group compared with the AKI and AKI+Cur-free groups.

cell injury in vitro, HK-2 human kidney tubular epithelial cells were treated with ascorbic acid-reduced myoglobin as described above (hereinafter referred to as the RM group). Myoglobin is a hemoprotein released from myocytes when RM occurs, which is one of the most important toxic factors leading to renal injury. As expected, the RM group exhibited significantly increased levels of lipid peroxidation products as measured by the MDA assay and obvious growth inhibition as measured by MTT assay (Fig. 3 A-B), suggesting successful establishment of RM-induced renal cell injury in vitro. In the drug treatment groups, HK-2 cells were treated with myoglobin in the presence of $50 \mu \mathrm{M}$ Cur-free or Cur-NP for $24 \mathrm{~h}$. The results revealed that both Cur-free and Cur-NP reduced the MDA levels induced by myoglobin (Fig. $3 \mathrm{~A}$ ), whereas the inhibition was more pronounced in the Cur-NP group compared with the Cur-free group. In addition, Cur-NP reversed the growth inhibition induced by myoglobin, 


\section{Cellular Physiology Cell Physiol Biochem 2017;43:2143-2154

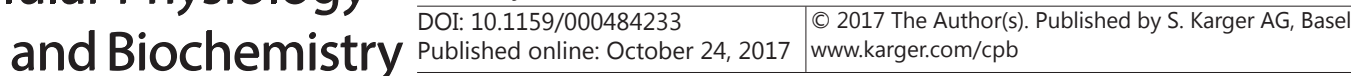 \\ Chen et al.: Curcumin-Loaded Nanoparticles Protect Against RM-Induced AKI}

which was not observed in the Cur-free group (Fig. 3 B).

Curcumin-loaded nanoparticles protected against myoglobin-induced cell apoptosis

The protective effect of Cur-free and Cur-NP on myoglobin-induced cell apoptosis was also examined. HK-2 cells were treated as described above. The cells were stained with Annexin-V and $\mathrm{PI}$, and subject to flow cytometry analysis to assess their apoptosis status. As shown in Fig. 4 A, the RM group exhibited obvious populations of early stage (Annexin- $\mathrm{V}^{+} \mathrm{PI}$ ) and late stage (Annexin- $\left.-\mathrm{V}^{+} \mathrm{PI}^{+}\right)$apoptosis (Fig. 4 A). Both Curfree and Cur-NP groups exhibited a marked decrease in Annexin-V and PI staining compared with the RM group (Fig. $3 \mathrm{~A}$ ). In addition, Cur-NP enhanced the protective effect against cell apoptosis compared with Cur-free, especially in the early stage of apoptosis (Annexin- $\mathrm{V}^{+} \mathrm{PI}$ ) when assessed at $24 \mathrm{~h}$ post injury (Fig. $4 \mathrm{~A}$ ). Caspase-3-mediated apoptosis in tubular cells is commonly observed in RM-induced renal injury $[14,15]$. Therefore, we next determined whether Cur-NP inhibited apoptosis by interfering with the Caspase-3 pathway. Western blot revealed that Cur-NP inhibited the myoglobin-induced up-regulation of apoptotic markers Caspase-3 and GRP-78 (Fig. 4 B-E) and was more effective than Cur-free. These results suggest a possible underlying anti-apoptosis mechanism of Cur-NP through down-regulation of Caspase-3 and GRP-78.

Curcumin-loaded nanoparticles protected against rhabdomyolysis-induced AKI in vivo

Given that the above data indicate that Cur-NP is more efficient than Cur-free in preventing RM-induced renal cell injury in vitro, we next investigated the efficacy of Cur-NP in treating RM-induced AKI in an animal model. The AKI model was established by injecting $50 \%$ glycerol at a single dose of $10 \mathrm{mg} / \mathrm{kg}$ in SD rats. Twenty-four h after the glycerol injection, rats in the treatment groups were intravenously injected with Cur-NP or Cur-free at a single dose of $10 \mathrm{mg} / \mathrm{kg}$. All mice were sacrificed $72 \mathrm{~h}$ post initial glycerol injection. Serum and renal tissues were collected and analyzed for the development of AKI. Kidney function tests indicated that glycerol injection resulted in a significant increase in serum $\mathrm{CPK}, \mathrm{Cr}$ and urea compared with the sham group. In the drug treatment groups, the administration of CurNP significantly reduced the serum levels of $\mathrm{CPK}, \mathrm{Cr}$ and urea. The reductions were not significant in the free Cur-free treatment group (Fig. $45 \mathrm{~A}-\mathrm{C}$ ). Furthermore, H\&E staining of renal sections revealed swelling and necrosis of tubular cells in the AKI group, whereas the renal tissues from the sham group appeared normal (Fig. 4 and 5 D). Cur-NP treatment reduced the histopathological damage in the AKI model; such improvement was not observed in the free Cur-free group (Fig. 4, $5 \mathrm{D}$ and Table 1). Collectively, these results demonstrated that Cur-NP could ameliorate the disease severity in AKI rats.

Curcumin-loaded nanoparticles relieved oxidative stress and reduced cell apoptosis in the AKI model

Given that in vitro data indicated that Cur-NP exhibits better anti-oxidative properties compared with Cur-free, we next determined whether Cur-NPs are more effective in relieving the oxidative stress in the AKI model. MDA assays revealed a marked increase in lipid peroxidation in renal tissues from glycerol-treated mice (hereinafter referred to as the AKI group), which is significantly inhibited by Cur-NP treatment (Fig. 6 A). However, no significant difference in lipid peroxidation was noted between the AKI group and Cur-free group (Fig. $6 \mathrm{~A}$ ). These data suggest that Cur-NP is more effective in relieving oxidative stress in vivo than Cur-free.

Given that the above in vitro data implicates a possible underlying anti-apoptosis mechanism for Cur-NP via down-regulation of Caspase-3 and GRP-78 (Fig. 4), we next 


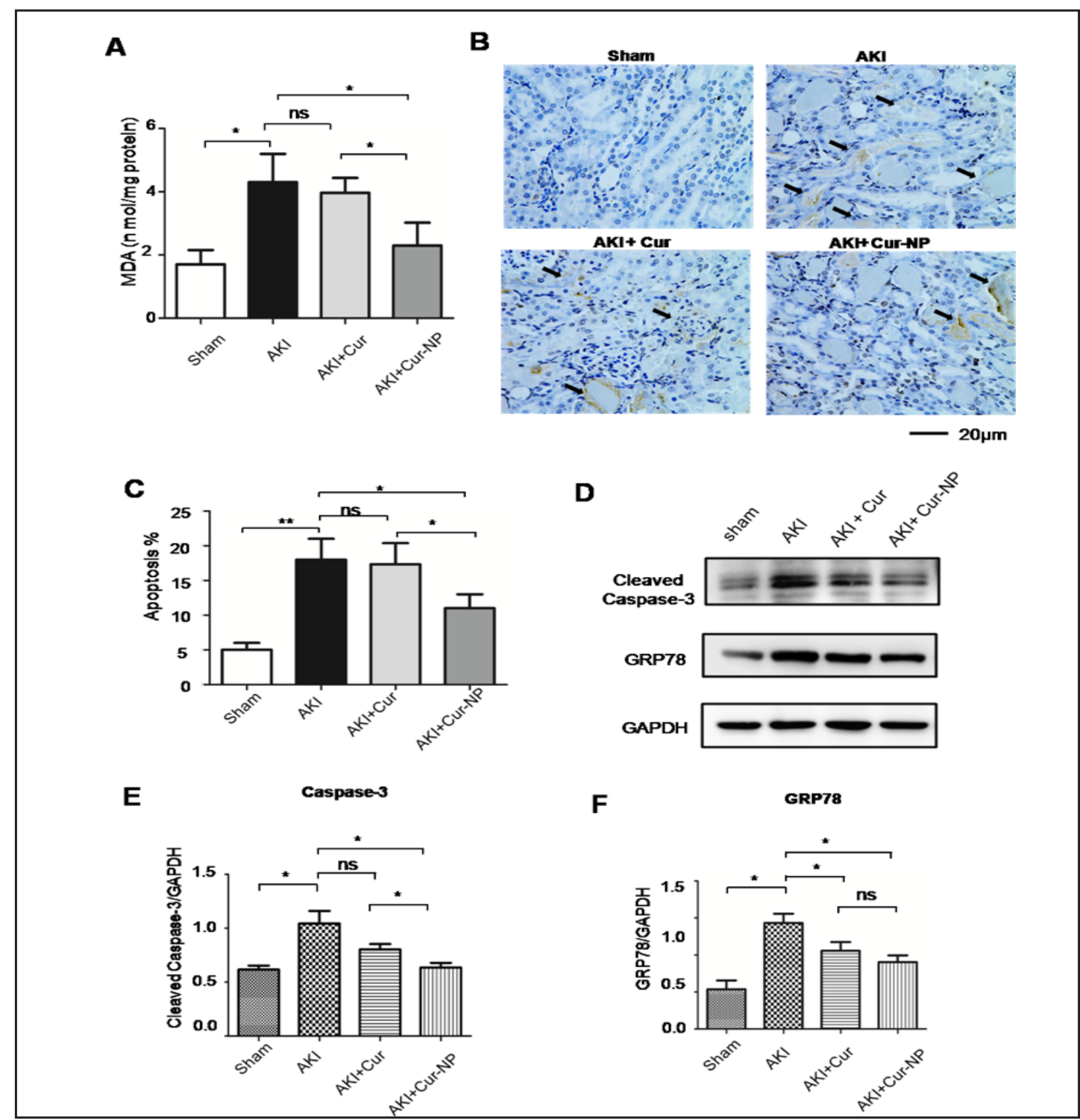

Fig. 6. Cur-NP relieves oxidative stress and reduces cell apoptosis in the glycerol-induced AKI model. (A) Lipid peroxidation levels as measured by MDA assays in kidney tissues of rats from all groups. Data are presented as the mean \pm SD of each group. (B) Representative photos of TUNEL-stained kidney sections from each group. Scale bar: $20 \mu \mathrm{m}$. Positive staining (See arrows) represents tubular epithelial cell apoptosis. (C) Photos of TUNEL-stained kidney sections of each rat ( $n=6 /$ group) were obtained from five random fields with 400x magnification and analyzed by Image-Pro Plus 6.0. The percentages of apoptotic cells were calculated as positive-stained cells/total cell numbers per field. Data are presented as the mean \pm SD of each group. (D) Western blot depicting caspase-3, GRP-78 and GAPDH protein expression in kidney tissues from all groups. (E) Quantitative analysis of Western blotting results. Specific protein expression levels were normalized to GAPDH levels. Data are presented as the mean \pm SD of three independent experiments. Statistical analysis between highlighted groups was determined by student's t-test. Significance levels were labeled as follows: $\mathrm{ns} \mathrm{P}>0.05$; $^{*} \mathrm{P}<0.05$; $^{* *} \mathrm{P}<0.01$.

determined whether Cur-NP exhibits similar anti-apoptosis effects in vivo. Renal tissues from different experimental groups were stained with TUNEL and analyzed for apoptotic cells. Consistent with previous studies, glycerol injection induced apoptosis in numerous tubular cells (Fig. 6 B). In the treatment groups, Cur-NP significantly reduced tubular cell 


\section{Cellular Physiology Cell Physiol Biochem 2017;43:2143-2154

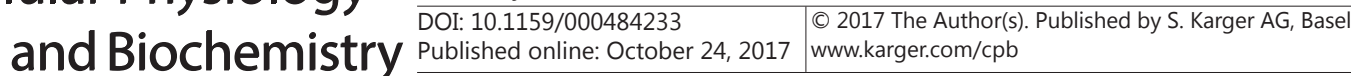 \\ Chen et al.: Curcumin-Loaded Nanoparticles Protect Against RM-Induced AKI}

apoptosis compared with the AKI group, whereas no such inhibition was observed in the Cur-free group (Fig. 6 B-C). Western blot assay revealed a decrease in cleaved Caspase- 3 and GRP-78 expression in the Cur-NP group compared with AKI group (Fig. 6 D-F). No inhibition was observed in the Cur-free group (Fig. 6 D-F). Taken together, these data support the hypothesis that Cur-NP exerts its enhanced protective effect in the AKI model through suppressing the apoptotic pathway, resulting in better treatment efficacy in AKI.

\section{Discussion}

RM is a clinical syndrome characterized by skeletal muscle fiber necrosis and the consequent release of intracellular contents [2]. Although the exact causes of RM are diverse, evidence supports a common pathway that leads to RM. Specifically, an increase in intracellular calcium concentrations is central to the destruction of myocytes and subsequent release of intracellular proteins. such as myoglobin, creatine kinase (CPK) and lactate dehydrogenase (LDH). Myoglobin is a hemoprotein and is one of the most important factors leading to renal injury by both direct and indirect mechanisms. A previous study and our data indicate that the oxidative state is essential for the cytotoxicity of myoglobin. The Fenton reaction $\left(\mathrm{Fe}^{2+}\right.$ to $\mathrm{Fe}^{3+}$; and $\mathrm{Fe}^{3+}$ to $\mathrm{Fe}^{4+}$ ) generates hydroxyl radicals $(\mathrm{OH})$ and causes radical-mediated injury in renal tubular cells $[7,16]$. Our study reported that reduced myoglobin (reduced by ascorbic acid) inhibited proliferation and induced apoptosis in renal proximal tubular HK-2 cells. In addition, Western blot assay revealed an increase in Caspase-3 and GRP-78 in cells treated with reduced myoglobin accompanied with an increase in lipid peroxidation as measured by the MDA assay. Taken together, our results confirmed the previous observation that myoglobin-induced oxidative injury is an important cause of cell apoptosis in rhabdomyolysis.

The clinical presentation of rhabdomyolysis varies greatly due to the wide range of causes $[1,2]$. AKI is a common complication of rhabdomyolysis, which is more severe when renal failure develops [7]. In the clinic, an increase in serum CPK levels is used to diagnose rhabdomyolysis, whereas elevated serum $\mathrm{Cr}$ and urea levels indicate kidney damage by rhabdomyolysis [2]. In this study, SD rats were intramuscularly injected with a single dose of $10 \mathrm{mg} / \mathrm{kg}$ glycerol as previously described [17, 18]. Glycerol-treated rats exhibited significantly elevated levels of serum $\mathrm{CPK}, \mathrm{Cr}$ and urea compared with control animals. Histologic changes, such as swelling and necrosis in tubular epithelial cells, and an increase in apoptotic cells were observed in renal tissues from glycerol-treated rats. In addition, MDA assays revealed enhanced generation of peroxidation products in renal tissues. Our data confirmed that one possible mechanism underlying AKI is the increase in cell apoptosis induced by excessive oxidative stress. Therefore, anti-oxidants represent potential therapeutics in treating rhabdomyolysis-induced AKI.

Curcumin, an active ingredient of turmeric, has potent anti-oxidant and radical scavenging properties [19-21]. Recent studies have demonstrated the therapeutic benefits of curcumin in several oxidative stress models, such as Alzheimer's disease and myocardial ischemia $[10,22]$. However, the main limitations for the successful use of curcumin as a therapy are its low aqueous solubility, poor absorption and high rate of metabolism [23, 24]. Recently, various nano formulations of curcumin have been synthesized to overcome these issues. Nanoparticle-loaded curcumin appeared to achieve high permeability, good bioactivity and low metabolism in animal models [25-27]. In the present study, the nanomaterial distearoylphosphatidyethanolamin-polyethylene glycol (DSPE-PEG) was employed as a nanocarrier for curcumin. The core-shell structure allows the poorly watersoluble curcumin to congregate in the DSPE, whereas PEG can reduce the clearance of cholesterol-free liposomal formulations and prolong its circulation time [28-30].

In the present study, Cur-NP inhibited the production of lipid peroxidation induced by myoglobin in HK-2 cells, suggesting an enhanced anti-oxidative effect compared with Curfree in vitro. In addition, Cur-NP treatment reversed the growth inhibition and cell apoptosis induced by myoglobin, and the protection effect was more pronounced compared with 


\section{Cellular Physiology Cell Physiol Biochem 2017;43:2143-2154 \begin{tabular}{l|l|l} 
DOI: 10.1159/000484233 & $\begin{array}{l}\text { O 2017 The Author(s). Published by S. Karger AG, Basel } \\
\text { www.karger.com/cpb }\end{array}$
\end{tabular}

Cur-free. Given that the greatest advantage of Cur-NP is its prolonged and constant release profile, the treatment benefit was more obvious in vivo. In the glycerol-induced AKI model, Cur-NP ameliorated disease severity as characterized by reduced serum $\mathrm{CPK}, \mathrm{Cr}$ and urea levels and less severe histological changes in renal tubular cells. Consistent with the in vitro results, reductions in Caspase-3 and GRP-78 were observed in the renal tissues from the Cur-NP group compared with the Cur-free group, whereas these levels are reduced in the AKI group. As the reduced lipid peroxidation product was observed in these renal tissues from the Cur-NP group, it is suggested that an increased anti-oxidative bioactivity of Cur-NP is responsible for the reduced cell apoptosis and thereby a less severe disease phenotype.

In conclusion, the present study investigated the efficacy and underlying mechanisms of Cur-NP in treating renal injury both in vitro and in vivo. The results collectively suggest that Cur-NP is a more effective anti-oxidant compared with Cur-free, resulting in the enhanced efficacy in treating rhabdomyolysis-induced renal injury both in vitro and in vivo. Furthermore, the enhanced protective effect of Cur-NP was more pronounced in the in vivo model compared with the in vitro experiment, and these results potentially benefited from its prolonged release behavior. Future studies are required to investigate other mechanisms that might be involved regarding how the cells and animals respond to Cur-NP, allowing for the development of more effective therapeutics for rhabdomyolysis-induced AKI.

\section{Acknowledgements}

We greatly appreciate Professors Zhong-cheng Yin, Chun Dai, Dong Sun and Sheng-kai Li from the Department of Nephrology of the Affiliated Huai,an Hospital of Xuzhou Medical University for technical assistance. This work was supported by a grant from the National Natural Science Foundation of China (No. 81670624).

\section{Disclosure Statement}

The authors declare that they have no conflicts of interest with the contents of this article.

\section{References}

1 Zutt R, van der Kooi AJ, Linthorst GE, Wanders RJ, de Visser M: Rhabdomyolysis: review of the literature. Neuromuscul Disord 2014;24:651-659.

-2 Bagley WH, Yang H, Shah KH: Rhabdomyolysis. Intern Emerg Med 2007;2:210-218.

-3 Tolesani Junior O, Roderjan CN, do Carmo Neto E, Ponte MM, Seabra MC, Knibel MF: Haff disease associated with the ingestion of the freshwater fish Mylossoma duriventre (pacu-manteiga). Rev Bras Ter Intensiva 2013;25:348-351.

4 Lafrance JP, Miller DR: Acute kidney injury associates with increased long-term mortality. J Am Soc Nephrol 2010;21:345-352.

5 Melli G, Chaudhry V, Cornblath DR: Rhabdomyolysis: an evaluation of 475 hospitalized patients. Medicine (Baltimore) 2005;84:377-385.

6 Chawla LS, Eggers PW, Star RA, Kimmel PL: Acute kidney injury and chronic kidney disease as interconnected syndromes. N Engl J Med 2014;371:58-66.

-7 Holt SG, Moore KP: Pathogenesis and treatment of renal dysfunction in rhabdomyolysis. Intensive Care Med 2001;27:803-811.

-8 Fu XY, Zhang DW, Li YD, Zhao PW, Tang YQ, Niu JZ, Li Y: Curcumin treatment suppresses CCR7 expression and the differentiation and migration of human circulating fibrocytes. Cell Physiol Biochem 2015;35:489498.

-9 Morabito R, Falliti G, Geraci A, Spada GL, Marino A: Curcumin Protects -SH Groups and Sulphate Transport after Oxidative Damage in Human Erythrocytes. Cell Physiol Biochem 2015;36:345-357. 


\section{Cellular Physiology Cell Physiol Biochem 2017;43:2143-2154 \begin{tabular}{ll|l} 
DOI: 10.1159/000484233 & $\begin{array}{l}\text { O 2017 The Author(s). Published by S. Karger AG, Basel } \\
\text { www.karger.com/cpb }\end{array}$
\end{tabular} \\ Chen et al.: Curcumin-Loaded Nanoparticles Protect Against RM-Induced AKI}

10 Manikandan P, Sumitra M, Aishwarya S, Manohar BM, Lokanadam B, Puvanakrishnan R: Curcumin modulates free radical quenching in myocardial ischaemia in rats. Int J Biochem Cell Biol 2004;36:19671980.

11 Ireson C, Orr S, Jones DJ, Verschoyle R, Lim CK, Luo JL, Howells L, Plummer S, Jukes R, Williams M, Steward WP, Gescher A: Characterization of metabolites of the chemopreventive agent curcumin in human and rat hepatocytes and in the rat in vivo, and evaluation of their ability to inhibit phorbol ester-induced prostaglandin E2 production. Cancer Res 2001;61:1058-1064.

12 Zheng D, Li X, Xu H, Lu X, Hu Y, Fan W: Study on docetaxel-loaded nanoparticles with high antitumor efficacy against malignant melanoma. Acta Biochim Biophys Sin (Shanghai) 2009;41:578-587.

$\checkmark 13$ Luo LN, Xie Q Zhang XG, Jiang R: Osthole decreases renal ischemia-reperfusion injury by suppressing JAK2/ STAT3 signaling activation. Exp Ther Med 2016;12:2009-2014.

14 Wang YD, Zhang L, Cai GY, Zhang XG, Lv Y, Hong Q, Shi SZ, Yin Z, Liu XF, Chen XM: Fasudil ameliorates rhabdomyolysis-induced acute kidney injury via inhibition of apoptosis. Ren Fail 2011;33:811-818.

15 Korrapati MC, Shaner BE, Schnellmann RG: Recovery from glycerol-induced acute kidney injury is accelerated by suramin. J Pharmacol Exp Ther 2012;341:126-136.

16 Zager RA: Mitochondrial free radical production induces lipid peroxidation during myohemoglobinuria. Kidney Int 1996;49:741-751.

17 Singh AP, Junemann A, Muthuraman A, Jaggi AS, Singh N, Grover K, Dhawan R: Animal models of acute renal failure. Pharmacol Rep 2012;64:31-44.

18 Sauriyal DS, Jaggi AS, Singh N, Muthuraman A: Investigating the role of endogenous opioids and KATP channels in glycerol-induced acute renal failure. Fundam Clin Pharmacol 2012;26:347-355.

-19 Ak T, Gulcin I: Antioxidant and radical scavenging properties of curcumin. Chem Biol Interact 2008;174:2737.

-20 Aftab N, Vieira A: Antioxidant activities of curcumin and combinations of this curcuminoid with other phytochemicals. Phytother Res 2010;24:500-502.

-21 Zhou Y, Zhang T, Wang X, Wei X, Chen Y, Guo L, Zhang J, Wang C: Curcumin Modulates Macrophage Polarization Through the Inhibition of the Toll-Like Receptor 4 Expression and its Signaling Pathways. Cell Physiol Biochem 2015;36:631-641.

22 Ringman JM, Frautschy SA, Cole GM, Masterman DL, Cummings JL: A potential role of the curry spice curcumin in Alzheimer's disease. Curr Alzheimer Res 2005;2:131-136.

23 Anand P, Kunnumakkara AB, Newman RA, Aggarwal BB: Bioavailability of curcumin: problems and promises. Mol Pharm 2007;4:807-818.

24 Yang W, Fu J, Yu M, Wang D, Rong Y, Yao P, Nussler AK, Yan H, Liu L: Effects of three kinds of curcuminoids on anti-oxidative system and membrane deformation of human peripheral blood erythrocytes in high glucose levels. Cell Physiol Biochem 2015;35:789-802.

25 Yallapu MM, Dobberpuhl MR, Maher DM, Jaggi M, Chauhan SC: Design of curcumin loaded cellulose nanoparticles for prostate cancer. Curr Drug Metab 2012;13:120-128.

-26 Lim KJ, Bisht S, Bar EE, Maitra A, Eberhart CG: A polymeric nanoparticle formulation of curcumin inhibits growth, clonogenicity and stem-like fraction in malignant brain tumors. Cancer Biol Ther 2011;11:464-473.

27 Li J, Wang P, Ying J, Chen Z, Yu S: Curcumin Attenuates Retinal Vascular Leakage by Inhibiting Calcium/ Calmodulin-Dependent Protein Kinase II Activity in Streptozotocin-Induced Diabetes. Cell Physiol Biochem 2016;39:1196-1208.

-28 Wang R, Xiao R, Zeng Z, Xu L, Wang J: Application of poly(ethylene glycol)-distearoylphosphatidyletha nolamine (PEG-DSPE) block copolymers and their derivatives as nanomaterials in drug delivery. Int J Nanomedicine 2012;7:4185-4198.

29 Nellis DF, Ekstrom DL, Kirpotin DB, Zhu J, Andersson R, Broadt TL, Ouellette TF, Perkins SC, Roach JM, Drummond DC, Hong K, Marks JD, Park JW, Giardina SL: Preclinical manufacture of an anti-HER2 scFvPEG-DSPE, liposome-inserting conjugate. 1. Gram-scale production and purification. Biotechnol Prog 2005;21:205-220.

-30 Zheng L, Li Y, Li X, Kou J, Zhong Z, Jiang Y, Liu Z, Tian Y, Yang L: Combination of Hydroxyl Acetylated Curcumin and Ultrasound Induces Macrophage Autophagy with Anti-Apoptotic and Anti-Lipid Aggregation Effects. Cell Physiol Biochem 2016;39:1746-1760. 